\title{
VIVENCIAS OCUPACIONALES DE PERSONAS RECIENTEMENTE JUBILADAS EN EL ENTORNO URBANO DE LA PROVINCIA DE A CORUÑA
}

\author{
OCCUPATIONAL EXPERIENCES OF RECENTLY RETIRED PEOPLE IN THE URBAN
} ENVINRONMENT OF THE PROVINCE OF CORUÑA

\section{María del Carmen Miranda-Duro' ${ }^{\text {, Marta Carracedo-Vázquez }}{ }^{2}$, Laura Nieto-Riveiro ${ }^{3}$, Betania Groba González ${ }^{4}$, Thais Pousada García ${ }^{5}$, Javier Pereira Loureiro ${ }^{6}$.}

\footnotetext{
1-6 Dirección postal: Universidade da Coruña, Grupo de Redes de Neuronas Artificiales y Sistemas Adaptativos - Informática Médica y Diagnóstico Radiológico (RNASA-IMEDIR), Instituto de Investigación Biomédica de A Coruña (INIBIC), Centro de Investigación en Tecnologías de las Informaciones y las Comunicaciones (CITIC), Facultade de Ciencias da Saúde, Oza, 15071 A Coruña, España.

1-6 Teléfono: 0034981167000 Ext. 5870
}

\footnotetext{
Grado en Terapia Ocupacional por la Universidade da Coruña. Terapeuta Ocupacional Investigadora. Universidade da Coruña, Grupo de Redes de Neuronas Artificiales y Sistemas Adaptativos - Informática Médica y Diagnóstico Radiológico (RNASA-IMEDIR), Instituto de Investigación Biomédica de A Coruña (INIBIC), Centro de Investigación en Tecnologías de las Informaciones y las Comunicaciones (CITIC), Facultade de Ciencias da Saúde. Correo electrónico: carmen.miranda@udc.es

Grado en Terapia Ocupacional por la Universidade da Coruña. Terapeuta Ocupacional Investigadora. Universidade da Coruña, Grupo de Redes de Neuronas Artificiales y Sistemas Adaptativos - Informática Médica y Diagnóstico Radiológico (RNASA-IMEDIR), Instituto de Investigación Biomédica de A Coruña (INIBIC), Centro de Investigación en Tecnologías de las Informaciones y las Comunicaciones (CITIC), Facultade de Ciencias da Saúde. Correo electrónico: marta.carracedo.vazquez@gmail.com

Doctorado en Ciencias de la Salud, Máster en Asistencia e Investigación Sanitaria y Grado en Terapia Ocupacional, por la Universidade da Coruña. Profesora Asociada. Universidade da Coruña, Grupo de Redes de Neuronas Artificiales y Sistemas Adaptativos - Informática Médica y Diagnóstico Radiológico (RNASA-IMEDIR), Instituto de Investigación Biomédica de A Coruña (INIBIC), Centro de Investigación en Tecnologías de las Informaciones y las Comunicaciones (CITIC), Facultade de Ciencias da Saúde. ResearcherID: N-7788-2013. ORCID ID: 0000-0003-3460-5895. Correo electrónico: Inieto@udc.es

4 Doctorado en Ciencias de la Salud, Máster en Asistencia e Investigación Sanitaria y Diplomatura en Terapia Ocupacional, por la Universidade da Coruña. Profesora Asociada. Universidade da Coruña, Grupo de Redes de Neuronas Artificiales y Sistemas Adaptativos - Informática Médica y Diagnóstico Radiológico (RNASA-IMEDIR), Instituto de Investigación Biomédica de A Coruña (INIBIC), Centro de Investigación en Tecnologías de las Informaciones y las Comunicaciones (CITIC), Facultade de Ciencias da Saúde. ResearcherID: K-1484-2016. ORCID ID: 0000-0001-5547-2337. Correo electrónico: bgroba@udc.es

Doctorado en Ciencias de la Salud, Máster en Asistencia e Investigación Sanitaria, Grado en Enfermería y Diplomatura en Terapia Ocupacional, por la Universidade da Coruña. Profesora Contratada Doctor. Universidade da Coruña, Grupo de Redes de Neuronas Artificiales y Sistemas Adaptativos - Informática Médica y Diagnóstico Radiológico (RNASA-IMEDIR), Instituto de Investigación Biomédica de A Coruña (INIBIC), Centro de Investigación en Tecnologías de las Informaciones y las Comunicaciones (CITIC), Facultade de Ciencias da Saúde. ResearcherID: N-9807-2016. ORCID ID: 0000-0003-3356-0429. Correo electrónico: tpousada@udc.es

6 Doctor en Informática y Licenciado en Informática, por la Universidade da Coruña. Profesor Titular de Universidad. Universidade da Coruña, Grupo de Redes de Neuronas Artificiales y Sistemas Adaptativos - Informática Médica y Diagnóstico Radiológico (RNASAIMEDIR), Instituto de Investigación Biomédica de A Coruña (INIBIC), Centro de Investigación en Tecnologías de las Informaciones y las Comunicaciones (CITIC), Facultade de Ciencias da Saúde. ResearcherID: D-5343-2012. ORCID ID: 0000-0001-9328-0723. Correo electrónico: javierp@udc.es
} 


\section{Resumen}

Objetivos: En la actualidad, se ha podido observar un envejecimiento progresivo de la población, lo que ha aumentado el interés acerca de la jubilación, ya que la duración de esta será cada vez más prolongada y afectará a los sistemas laborales, sanitarios y educativos, así como a la propia organización de la sociedad. El objetivo de este estudio es explorar y conocer las vivencias ocupacionales de los participantes en su etapa de jubilación.

Método: Se ha utilizado la investigación cuantitativa y cualitativa, empleándose como métodos de recogida de datos un cuestionario, una entrevista semiestructurada y un diario de campo. La muestra estuvo conformada por 13 personas jubiladas.

Resultados: Existen inconsistencias entre los resultados obtenidos en el cuestionario y lo que los participantes refieren en la entrevista, ya que en el cuestionario otorgan a la jubilación un significado positivo mientras en sus discursos se refleja lo contrario. Además, presentan limitaciones para comprender el concepto oficial de jubilación e identificarse con él. Por último, ha destacado la influencia del clima en el desempeño ocupacional y la controversia existente en cómo se entiende el ocio según el entorno en el que viva la persona (urbano o rural).

Conclusiones: Se concluye que la distribución del tiempo es uno de los cambios más significativos de la jubilación, existiendo contradicciones en cuanto al significado que se le otorga a este concepto. Los resultados obtenidos también reflejan la necesidad de la preparación previa a la jubilación, aunque inicialmente muchos participantes no identifiquen esta necesidad como propia.

\section{Palabras clave}

jubilación, envejecimiento, terapia ocupacional, población urbana.

\section{Abstract}

Aims: Nowadays has been observed a progressive aging of the population which has increased the interest about retirement, as the duration of this will be longer and will affect labour, health and education systems, as well as the proper organization of society. The objective of this study is to explore and learn about the occupational experiences of older recently retired in the urban environment of the province of A Coruña.

Methods: It has been used for the study the quantitative and qualitative research, being used as methods of data collection a questionnaire, a semi-structured interview and a field journal homemade. The sample consisted of 13 retired people.

Results: There are inconsistencies between the results of the questionnaire and refer participants in the interview, because the retirement questionnaire give a positive meaning while in his speech is the opposite. Furthermore, they have limitations to understand the official concept of retirement and identify with him. Finally, it highlighted the influence of climate on occupational performance and the controversy about how leisure is understood as the environment in which the person lives (urban or rural).

Conclusions: We conclude that the distribution of time is one of the most significant changes in retirement, existing contradiction about the meaning given to it. The results also reflect the need for pre-retirement preparation, although initially many participants do not identify this need as their own.

\section{Keywords}

retirement, aged, occupational therapy, urban population.

Recepcionado: 08/11/2016

Aceptado: 30/05/2017 


\section{INTRODUCCIÓN}

En el panorama actual de la sociedad, principalmente en la Unión Europea y Japón, se observa un incremento de la esperanza de vida. Dicha situación junto con la baja natalidad da lugar que con el transcurso del tiempo se produzca un envejecimiento progresivo de la población. Este fenómeno social había sido observado aunque actualmente el envejecimiento se ha presentado de forma especialmente acelerada (HelpAge International, 2015; United Nations, 2015).

Debido a este fenómeno, la jubilación se está convirtiendo en uno de los temas de mayor interés para la sociedad, tanto a nivel de abordaje como de investigación. Por ello, si aumenta la esperanza de vida, la etapa de jubilación tendrá una duración más prolongada, adquiriendo así una mayor importancia en cuanto a los sistemas laborales, sanitarios y educativos, como también en la propia organización de la sociedad (Abellán \& Pujol, 2015).

La jubilación se corresponde con el final de la vida laboral de una persona, pudiendo realizarse de forma voluntaria o forzosa. La diferencia se centra en respetar el derecho de la persona de elegir cuando jubilarse (Aymerich, Planes \& Gras, 2010). En cada país hay una edad establecida para jubilarse existiendo diferentes tipos, tal y como se refleja en Ley de la Seguridad Social sobre Jubilación (2011) en el caso de España.

La jubilación es la etapa de la vida considerada como aquella en la que más cambios se sufren tras cesar la vida laboral. En relación con esto, Atchley (1975) ha definido un proceso de adaptación a la jubilación, que presenta las siguientes etapas: etapa de prejubilación que se corresponde con las expectativas ambiciosas de cara al cese laboral, etapa de jubilación que se caracteriza por una época de euforia y liberación o también como la continuidad de las actividades de ocio realizadas anteriormente, etapa de desencanto que se corresponde con una percepción de la realidad que no era como se había esperado provocando estado anímico bajo, etapa de reorientación que da lugar cuando la persona se sitúa en términos más realistas y por último, etapa de estabilidad, que es cuando la persona finalmente asume una visión realista teniendo en cuenta las posibilidades y limitaciones relacionadas con la jubilación. (Aymerich et al., 2010).

Por otro lado, es interesante mencionar el Modelo Transteórico de Prochaska y Diclemente (1984) que las autoras Leandro, Giardini y Bratfisch (2014) adaptan al proceso de preparación para la jubilación en los que se distinguen las siguientes etapas: pre-contemplación, contemplación, preparación, acción y mantenimiento; de manera que, la persona en un primer lugar ignora o no tiene interés por planificar su jubilación, pasa a ser consciente de las dificultades que tendrá que enfrentar, empieza a plantearse ideas de cara a futuro, lleva a cabo esos planes y por último, se consolidan los logros alcanzados.

En relación con lo anterior, surge en las Jornadas Internacionales Ilevadas a cabo por la Unión Democrática de Pensionistas y Jubilados (UDP) de España (1990), por primera vez el tema de la preparación para la jubilación, transmitiendo la importancia de la creación de programas en una etapa previa al cese laboral.

Los programas de preparación para la jubilación de la UDP concuerdan con algunas de las líneas centrales del Marco del Envejecimiento Activo, ya que en ambos se muestra la importancia de aprender a lo largo de la vida, reflejándose que es tan importante aprender un oficio para desempeñar la vida laboral, como aprender a salir de la rutina del trabajo y continuar con un estilo de vida diferente. Como bien se nombraba anteriormente en la jubilación surgen cambios, por lo que es entendida como un momento de transición, existiendo diferentes tipos de mecanismos de adaptación según la persona. (Centro Internacional de Longevidad de Brasil, 2015)

Desde la perspectiva de la Terapia Ocupacional (TO) la jubilación es un proceso que implica la transición de ocupaciones, esto hace referencia a un cambio de etapa a otra, y en dicho cambio las personas emplean diferentes mecanismos de adaptación que les permiten ajustarse a una nueva rutina, generándose cambios en los roles desempeñados por la persona. (Pettican \& Prior, 2011; Jonsson, Josephsson \& Kielhofner, 2001)

En relación con la transición ocupacional, también es relevante mencionar la identidad ocupacional. Según Christiansen (1999), la participación en la ocupación contribuye a construir la identidad y es el principal medio para comunicar la identidad de uno.

El trabajo o productividad se entiende como una actividad que ha adquirido significado para la persona en situación de jubilación, la que ha ocupado la mayor parte de su vida. Por lo tanto, entendiendo el trabajo como una ocupación, este contribuye a crear la identidad ocupacional de las personas, dando sentido y significado a sus vidas, generando bienestar y a su vez, calidad de vida. De manera que si el trabajo es la ocupación a la que más tiempo se le dedica en el transcurso de la vida de una persona, se generarán cambios en cuanto a la identidad ocupacional y por consiguiente, en el 
desempeño ocupacional, por lo que sería necesaria la intervención de terapeutas ocupacionales.

Finalmente se cree necesario explorar las experiencias de cada persona en esta etapa de transición desde la perspectiva del envejecimiento activo, analizando los cambios que se producen en la transición del trabajo a retiro conociendo a su vez si estos cambios afectan a la salud y a la calidad de vida; conocer el significado que las personas jubiladas le otorgan a la propia jubilación, e indagar sobre la necesidad de una preparación previa a esta etapa.

\section{MÉTODO}

Este estudio se ha llevado a cabo a través de la investigación combinada, haciendo uso de la investigación cuantitativa siendo un estudio no experimental, transversa y descriptivo; por otro lado, la investigación cualitativa siendo un estudio de fenomenología descriptiva. Además, se ha centrado en tres ayuntamientos del entorno urbano de la provincia de A Coruña.

Para la selección de la muestra se ha empleado el muestreo teórico (Flick, 2015). En la Tabla I se pueden consultar los criterios de inclusión y exclusión establecidos para el presente estudio.

Finalmente se ha obtenido una muestra de 13 participantes codificados de P1 a P13, con una media de edad de 68 años $( \pm 4.74)$.

Como técnicas de recogida de datos se han utilizado: un cuestionario (Apéndice 1: Cuestionario), una entrevista semiestructurada (Apéndice 2: Guion de entrevista), y un diario de campo, todos de elaboración propia.

Para el análisis cuantitativo, se hizo uso del programa estadístico SPSS versión 24. Por un lado, se hizo un análisis descriptivo de las diferentes frecuencias a destacar. Por otro lado, se utilizó la prueba Chi-Cuadrado para estudiar la asociación entre variables o datos categóricos (Gerrish \& Lacey, 2008). Se estableció la significación estadística en $\mathrm{p}<0.05$.

Para el análisis cualitativo, se realizó una transcripción exacta de las entrevistas, codificando y categorizando posteriormente los discursos de los participantes (Flick 2015).

Finalmente, en este trabajo de investigación se han Ilevado a cabo una serie de procedimientos éticos. En primer lugar, se elaboró una hoja de información para los participantes, con el respectivo consentimiento informado, explicando con detalle en qué consiste su participación y resolviendo posibles dudas.
Del mismo modo, los participantes han dado su consentimiento para la publicación de los resultados del presente estudio.

Por otro lado, las entrevistas han sido grabadas y transcritas, anonimizando los datos para que no se pueda identificar a los participantes, destruyendo dichas grabaciones una vez que ya no fueron necesarias. Se respetó en todo momento la confidencialidad de los datos personales, bajo la Ley Orgánica 15/1999, de 13 de diciembre, de Protección de Datos de Carácter Personal.

\section{RESULTADOS}

\section{Resultados de la investigación cuantitativa}

A continuación se presentan los resultados obtenidos a través del cuestionario: en primer lugar, se mostrarán las frecuencias de las variables y, a continuación, diferentes asociaciones entre ellas. Cabe destacar que no es posible extraer inferencias o conclusiones definitivas, debido al reducido tamaño de la muestra.

Tal y como se ha mencionado, la muestra está compuesta por 13 personas, de las cuales 6 son hombres y 7 son mujeres. El participante más joven tiene 59 años, mientras que el más mayor tiene 74 , obteniéndose una media de edad de 68 años $( \pm 4.74)$. En cuanto al estado civil se obtiene que el $53.8 \%$ de los participantes están casados, un $15.4 \%$ están separados o viudos y un $7.7 \%$ divorciados y solteros. Además, destaca que el 39\% tienen estudios básicos.

Los participantes presentan diversidad de profesiones siendo 3 autónomos y el resto teniendo variabilidad de profesiones pertenecientes a los sectores de producción secundario y terciario. En cuanto a la duración de la vida laboral de la muestra se obtiene una media de 41 años, siendo la mínima de 30 y máxima de 51. Por otro lado, en relación con la duración de la jubilación se obtiene una media de 4.7 años $( \pm 2.7)$, mientras que la moda en torno a la duración de la jubilación es de 7 años.

Las personas que participaron en el estudio refieren estar muy satisfechos $(62.2 \%)$ o satisfechos $(30.8 \%)$ con el trabajo, no existiendo el caso de ni satisfecho ni insatisfecho o nada satisfecho. Además, es de destacar que únicamente una persona refiere haber realizado una preparación previa a la jubilación, obteniéndose por otro lado, que los participantes otorgan a la jubilación un significado positivo $(61.5 \%)$ o ni positivo ni negativo $(38.5 \%)$, no existiendo un significado negativo hacia esta etapa. 
Por otro lado, destaca que el $61.5 \%$ de los participantes dedican más tiempo al ocio y tiempo libre, frente al 7.7\% que lo dedican a la participación en la comunidad; en relación a la actividad a la que menos tiempo dedican, destaca el trabajo con un $46.2 \%$, frente a un $7.7 \%$ que identifica el descanso y sueño.

Se ha estudiado la asociación entre el sexo de los participantes y las siguientes variables: tipo de jubilación, carácter de la jubilación, satisfacción con la etapa laboral, significado de la jubilación y problemas de salud. $\mathrm{Al}$ analizar estas relaciones, se ha observado una fuerte asociación entre el sexo y el significado de la jubilación, siendo más frecuente en los hombres una valoración positiva de este proceso.

Los resultados obtenidos no permiten establecer que exista asociación entre el "sexo" y "tipo de jubilación". No obstante, cabe destacar que el $15.4 \%$ de las personas con una jubilación anticipada son hombres frente a un $30.8 \%$ de mujeres; mientras que el caso de la jubilación ordinaria es a la inversa, tal y como se muestra en la Figura 1.

Otra asociación estudiada a destacar ha sido entre "tipo de jubilación" y "problemas de salud", reflejándose en la Figura 2 que las personas que se han jubilado de forma ordinaria con un $38.5 \%$ tienen algunos problemas de salud y con un $15.4 \%$ ningún problema. Por otro lado, las personas jubiladas de forma anticipada presentan muchos problemas de salud, algunos y ningunos con un $15.4 \%$ cada uno.

Se ha estudiado la relación entre la variable "carácter de la jubilación" y las siguientes: satisfacción con la etapa laboral, significado de la jubilación y problemas de salud.

En relación con las asociaciones estudiadas, destaca la relación entre "carácter de la jubilación" y "significado de jubilación" ( $p=0.053)$. El 30.8\% de las personas que se han jubilado forzosamente entienden la jubilación como algo ni positivo ni negativo, mientras que el 15.4\% dice que es positiva. En cuanto a las personas que se han jubilado de forma voluntaria el $46.2 \%$ dice que la jubilación es positiva frente a un $7.7 \%$ que dice que ni es positiva ni negativa. Por lo tanto, se puede decir que el hecho de jubilarse de forma voluntaria se relaciona en mayor medida con una jubilación positiva, mientras que jubilarse de forma forzosa parece vincularse con un significado más negativo sobre la jubilación.

En un principio se habían realizado las asociaciones pertinentes entre la distribución del tiempo con: significado de jubilación, calidad de vida y problemas de salud. Ninguna de las asociaciones ha sido válida, pero como en casos anteriores, se cree necesario destacar algunas de ellas. En este caso entre "satisfacción con el tiempo" y "calidad de vida", observándose en la Figura 3 que de las personas que consideran que tienen buena calidad de vida el $76.9 \%$ están satisfechos con su distribución del tiempo frente al $15.4 \%$ que está muy satisfecho; mientras que las personas que consideran que no tienen buena calidad de vida no están nada satisfechos con la distribución de su tiempo.

En cuanto a la relación entre "satisfacción con la distribución del tiempo" y "estado civil" se refleja que los participantes casados están "satisfechos" o "muy satisfechos", frente a los no casados que están "nada satisfechos" o "satisfechos".

\section{Resultados de la investigación cualitativa}

Tras el análisis de las entrevistas realizadas y del diario de campo, han emergido un conjunto de categorías que favorecen la comprensión del fenómeno de estudio. En la Figura 4 se muestra un mapa conceptual de los diferentes niveles que las categorías adquieren según la relevancia, aunque es importante matizar que a pesar de existir una jerarquía en cuanto a la importancia que adquieren las categorías, todas están interrelacionadas. Se observa que Jubilación versus vejez es la categoría principal de la que parten las demás categorías, obteniendo mayor relevancia las Percepciones sobre la preparación para la jubilación, Profesión como factor determinante y Cambios producidos en los roles y ocupaciones. Además, se observa que de esta última categoría parten la Ambigüedad temporal, la Transición en el ocio y las Dificultades para el desempeño del ocio, ya que son factores que influyen directamente en dichos cambios.

\section{Jubilación versus vejez}

Cada persona ha vivido la transición de la vida laboral al retiro de forma diferente dependiendo del tipo de jubilación y de las redes de apoyo, por lo que la jubilación ha adquirido un significado diferente. Se ha podido observar que los participantes tienen en común la percepción negativa hacia la etapa de la jubilación. De acuerdo con lo anterior, ha resaltado lo mencionado cuando se le pregunta a la persona acerca de cuáles eran sus expectativas sobre la jubilación y cuáles son sus proyectos de futuro. Mientras estaban en su etapa laboral la mayoría refiere no haberle dado importancia al hecho de jubilarse ya que lo veían muy lejano. 
En cuanto a los proyectos de futuro dicen no tener o simplemente no ser capaces de identificarlos. Además no tienden a pensar de cara al futuro porque viven el presente. "Expectativas... vivir lo mejor posible. Porque otra cosa... Lo que me quede de jubilación tratar de vivi lo mejor posible, sin discusiones..." (P5). Por otro lado, resalta la preocupación general sobre los posibles problemas de salud y que estos limiten su independencia.

En cuanto a calidad de vida y relacionado con la jubilación, resalta el tema económico para llevar una vida digna, que está estrechamente relacionada con una buena calidad de vida.

\section{Cambios producidos en los roles y ocupaciones}

La jubilación es una etapa en la que los roles de las personas cambian, ya que pasan de tener un rol de trabajar a adquirir otros nuevos según las circunstancias de cada persona, además de desempeñar otro tipo de ocupaciones diferentes, aunque en algunos participantes admiten no apreciar un cambio.

El principal cambio que se observa en los participantes es la distribución del tiempo y que se han tenido que ir adaptando al cambio de rol de trabajador y de la rutina del trabajo, a tener que buscar otras cosas. "[...] O sea cambió algo en mi vida, iclaro!, la distribución del tiempo, porque tengo un tiempo que antes no tenía, y ahora resulta que tengo el tiempo tan ocupado como antes lo tenía, prácticamente. Con otras cosas claro (risa)" (P1).

Asimismo, al estar jubilados, también se observa que se dedican al cuidado de otros o que han tenido que dejar de trabajar para cuidar a otros, adquiriendo roles diferentes.

Relacionado con el cambio de roles y ocupaciones, el hecho de mantener o no las amistades que se habían establecido en el ámbito laboral también supone un cambio importante para esta etapa de la jubilación, ya que los amigos son una de las redes de apoyo principales para una persona.

\section{Transición del ocio}

La transición implica cambios y en el caso de los participantes se han observado cambios en cuanto a ocio que estos desempeñaban en su etapa laboral en comparación con la etapa actual.

Los participantes al preguntarles sobre qué actividades de ocio realizaban en su etapa laboral, coinciden en que durante esta etapa la carga horaria producida por la dedicación a su trabajo, no les permitía dedicar el tiempo suficiente al desempeño de dichas actividades."[... No tenía tiempo y los fines de semana era cuando más trabajaba. Siempre tenía que estar trabajando, si querías conseguir algo tenías que trabajar, no se podía andar por ahí. Al tener un negocio es lo que hay" (P8).

Asimismo, si realizaban actividades de ocio eran los fines de semana y principalmente acompañados de su familia, o simplemente no las realizaban por falta de tiempo libre. Las actividades que más mencionan son: viajar, leer, pasear y jugar a las cartas. En la mayoría de los casos continúan realizando dichas actividades. "[...]

Hago las mismas cosas, es más, intento, aunque ahora estoy en una mala época, pero voy al gimnasio, y ando más que antes y bien, no sabes. Es diferente. Es que antes el horario no me daba para más. "[...] Y bueno, me dedico mucho a la lectura [...]" (P2).

Se ha observado que los participantes tienen dificultades para identificar las actividades de ocio que realizan debido a que cuando se les pregunta sobre dichas actividades no las nombran todas pero sí durante su discurso.

\section{Dificultades para el desempeño del ocio}

A través del discurso de los participantes emergen diferentes limitaciones en cuanto a realización de actividades de ocio, algunos participantes dedican su tiempo libre al cuidado de otros, mientras que podrían dedica ese tiempo desempeñando actividades de ocio que estos considerasen preciso.

En uno de los casos, un participante hace referencia a que tiene que dedicar tiempo al hogar, por lo que esta puede ser identificada como una limitación para el desempeño óptimo del ocio.

Por otra parte, una de las limitaciones observadas es el hecho de que en el caso de algunos hombres que quieren dedicarse al trabajo en el campo como una actividad del ocio pero se ven limitados por sus mujeres, debido a que estas no se sienten atraídas por el entorno rural. "A ver a mí me gusta mucho el campo. [...] No se puede por el asunto familiar, porque a la mujer no le gusta entonces te tienes que quedar aquí... Escaparse un día o dos pero es lo que hay" (P5).

Además, algunos participantes encuentran limitaciones relacionadas con la salud por haber superado recientemente una enfermedad, por problemas visuales o problemas físicos. 


\section{Ambigüedad temporal}

La categoría de ambigüedad temporal hace referencia a que han emergido de los discursos dos temas en relación con el tiempo. Por una parte el clima, y por otra parte la distribución del propio tiempo. En cuanto al clima, algunos participantes mencionan que deciden qué actividades desempeñar cada día dependiendo del tiempo meteorológico, sea frío o calor. "Pues depende si es invierno, verano. Si hace mucho frío y no tengo mucho que hacer, ¿para qué voy a levantarme temprano? Si hace buen tiempo, ¿para qué voy a pasar tanto tiempo en cama? [...]" (P5).

La otra concepción del tiempo que ha resaltado en las entrevistas, es que emerge la idea de que no tienen la misma distribución del tiempo actualmente en comparación con la etapa laboral, ya que afirman poder realizar las actividades con mayor calma. Aunque en algunos casos estos tienen la percepción de que les hace falta más tiempo, porque mencionan estar más ocupados/as que en su etapa laboral.

\section{Profesión como factor determinante}

La profesión en este caso se entiende como aquella actividad laboral de carácter remunerado que se ha ejercido un cierto tiempo. Se entiende como categoría ya que en relación con la profesión han emergido diferencias entre el tipo de trabajo realizado y el tipo de relaciones sociales en el puesto laboral, como también el modo de acceder a la jubilación. Por otra parte, la elección de la profesión sea por vocación u otras circunstancias, puede haber influido en cómo la persona vive la jubilación y por lo tanto es de interés para el estudio realizado.

Se puede observar que las jubilaciones son anticipadas cuando es la empresa la que accede a que se jubile a la persona, o bien por motivos de salud. En otras ocasiones la decisión es de la persona cuando su pareja también se jubila.

En relación con la elección de la profesión sea por vocación o por otras circunstancias, resalta uno de los casos ya que es la única persona que cuando se le pregunta sobre su profesión habla en primera persona. "[...] elegí mi profesión de farmacéutica porque en mi casa me decían que era una profesión muy cómoda para mí, que yo el día de mañana podía tener mi farmacia y yo tenía mi vida resuelta y tranquila ¿no? Sin problemas. [...]" (P1).

\section{Percepciones sobre la preparación para la jubilación}

La preparación para la jubilación es entendida como aquellos programas destinados a la formación de las personas para su etapa de jubilación.

En cuanto a la preparación previa al cese laboral resalta el tema económico como algo que los participantes dan importancia en relación con esta etapa, de manera que, para tener una buena jubilación es importante tener una buena pensión. Además, se concibe el tema económico como un tipo de prevención para disfrutar de una vida digna.

Al preguntarles a los participantes si creen necesaria una preparación previa para la jubilación, no creen que ellos la necesiten, ya que únicamente observan la necesidad de dicha preparación en terceras personas por el hecho de que afirman que el trabajo no condicionaba sus vidas.

Además, en muchos casos no identifican la necesidad de prepararse previamente a la jubilación, ya que es considerado como algo normal y que todo el mundo debería de tener en cuenta. Esto puede ser debido a que la mayoría de ellos refiere haberse adaptado completamente a la etapa de la jubilación.

Es importante resaltar que se ha detectado que aunque no se afirme directamente una necesidad para estar preparados o concienciados de cómo va a ser la jubilación, se observa que realmente se desconoce quién puede ofrecer información o formación sobre dicha preparación.

Al final de la entrevista, se pregunta por segunda vez si se considera necesaria la preparación para la jubilación. En la mayoría de los casos llegan a la conclusión que sí que es necesaria, pero no se ven identificados en esa necesidad. En otros casos se mantiene la idea de que no es necesaria porque la jubilación es considerada como algo natural. "A ver, es irse haciendo a la idea que te va a cambiar la vida. No te viene de repente, tienes que pensar que esa situación se va a dar" (P13). 


\section{DISCUSIÓN}

Este trabajo se planteó con el objetivo principa de explorar y conocer las vivencias ocupacionales de personas recientemente jubiladas en el entorno urbano de la provincia de A Coruña. Los resultados obtenidos permitieron alcanzar tanto los objetivos generales como específicos marcados al comienzo del trabajo, los cuales se discuten a continuación

El cambio más significativo que ha destacado en el discurso de los participantes ha sido en relación a la distribución del tiempo, ya que la mayoría de ellos coincide en que este ha aumentado considerablemente tras el cese de la vida laboral. Estos resultados coinciden con los de otros autores, como es el estudio realizado por Allan, Wachholtz y Valdés (2005). Dichos autores obtienen que tanto los hombres como las mujeres refieren tener mayo libertad y tiempo, pudiendo realizar las actividades de forma más relajada.

Los informantes del presente estudio pertenecen a entorno urbano, por lo que han tenido que buscar otras actividades para ocupar el tiempo tras el proceso de transición vivido para retirarse. Esto está estrechamente relacionado con la teoría de la actividad y el envejecimiento activo y del mismo modo, forma parte de la transición ocupacional descrita por Christiansen (1999) y de las etapas de la adaptación definidas por Atchley (1975).

Asimismo, tal y como reflejan los resultados de investigación cuantitativa, los participantes dedican más tiempo en la actualidad a las actividades de ocio y tiempo libre, mientras que en el estudio de Hermida, Tartaglin y Stefani (2016) se refleja que los participantes otorgan mayor importancia al descanso, comienzo o continuidad.

En este sentido, en el estudio realizado por Pettican Prior (2011) se refleja la importancia de establecer un rutina satisfactoria en la jubilación, lográndose a través de la variedad de ocupaciones significativas a las que puedan acceder y participar, aspecto que coincide con los hallazgos encontrados en la presente investigación sobre la rutina diaria. Por lo tanto, se avala la importancia de intervenir desde terapia ocupacional a través de actividades con significado para las personas jubiladas, asegurando un adecuado equilibrio entre ellas.

En relación con los cambios percibidos en cuanto a roles, los participantes asumen que ya no desempeñan el rol de trabajadores, de modo que la etapa laboral pasa a un segundo plano. En contraposición a estos resultados, a autora Teuscher (2010) refiere que la pérdida del rol de trabajador tras el cese laboral no conllevó en los participantes una pérdida de la identidad profesional. En este caso, únicamente se ha percibido en un participante que no ha perdido su identidad profesional, al igual que ha observado Price (2002) que las mujeres con una actividad laboral remunerada experimentan un mayor sentido de pérdida al retirarse de su carrera en comparación con las mujeres que no tienen una actividad remunerada.

Como se ha mencionado en los resultados, la categoría de jubilación versus vejez está presente de forma transversal en el resto de resultados de la investigación, ya que la percepción sobre las personas mayores influye en cómo estas se perciben a sí mismas y el significado que otorgan a la jubilación. Con respecto a lo anterior es importante señalar que existen inconsistencias en el significado que otorgan a la jubilación en las entrevistas, en contraposición con lo que reflejan a través de cuestionario. De este modo, en el cuestionario otorgan a la jubilación un significado positivo mientras en sus discursos se refleja lo contrario.

De igual modo se han encontrado ciertas inconsistencias en las entrevistas, ya que los participantes no identifican como propia la necesidad de preparación previa a la jubilación. Sin embargo, al ahondar en sus discursos, se observa que sí existen ciertas necesidades que podrían abordarse en este tipo de programas, tal y como se ha reflejado en el apartado de resultados. Estas necesidades se vinculan principalmente con la modificación de percepciones negativas que presentan en torno al proceso de envejecimiento y la jubilación.

Cabe destacar que los participantes del presente estudio tienen dificultades para comprender el concepto de jubilación y a su vez, no se sienten identificados con dicho término. Esto puede ser debido a que como mencionan en sus resultados Vianna y Melo-Silva (2009, p. 46), se comprende que un jubilado es "como un solo hombre de edad que debe ser reemplazado por el nuevo, como algo ya utilizado y ahora tiene más espacio en el mundo de la producción".

Asimismo, se observa que los informantes no son capaces de identificar proyectos de cara al futuro, ya que se centran en vivir en el presente. Tienen una especial preocupación sobre los posibles problemas de salud que puedan estar por llegar, lo que puede limitar su nive de independencia, ya que relacionan la salud y calidad de vida con aspectos físicos y mentales. Asimismo, en hallazgos del estudio de Jonsson, Borell y Sadio (2003) se identifica que los participantes desean tener una estabilidad de cara al futuro en relación con su salud.

Por otra parte, en los resultados de este estudio se muestra la influencia que puede tener el clima en el desempeño ocupacional de los participantes a la hora 
o no de participar en una actividad. Por el momento no se ha encontrado evidencia que refuerce o contradiga este hallazgo, por lo que se considera que es necesario llevar a cabo futuras investigaciones que profundicen en esta relación.

Para finalizar cabe señalar que este estudio presenta una serie de limitaciones que impiden establecer conclusiones definitivas, como han sido las dificultades de acceso al campo y el reducido tamaño muestral en cuanto a la representatividad de los datos cuantitativos.

Por ello se cree necesario seguir investigando en el futuro sobre el papel de la terapia ocupacional en la preparación para la jubilación, estudiando si existen diferencias entre ámbito urbano y rural, y ampliar tanto el tamaño muestral como el ámbito de estudio.

\section{AGRADECIMIENTOS}

Este estudio se realizó en el marco de Trabajo Fin de Grado de la titulación de Grado en Terapia Ocupacional de la Universidade da Coruña.

Se agradece la colaboración de todas aquellas personas que han participado en este estudio tanto de forma directa como indirecta, ya que, sin la implicación de todas ellas, este trabajo no podría haberse llevado a cabo.

Se agradece asimismo el apoyo de la Xunta de Galicia a través de las ayudas para la consolidación y estructuración de unidades de investigación competitivas del Sistema Universitario de Galicia 2014 y 2012, parcialmente cofinanciadas por el Fondo Europeo de Desarrollo Regional (FEDER) de la Unión Europea: Grupo RNASA-IMEDIR y Agrupación Estratégica del Centro de Investigación en Tecnologías de la Información y las Comunicaciones (CITIC). Ref.GRC2014/049, CN2012/211.

\section{REFERENCIAS BIBLIOGRÁFICAS}

Abellán, A. \& Pujol, R. (2015). Un perfil de las personas mayores en España. Indicadores estadísticos básicos. Informes Envejecimiento en red $\mathrm{n}^{\circ} 10$. Recuperado de http://envejecimiento.csic.es/documentos/ documentos/enred-indicadoresbasicos15.pd

Allan, N., Wachholtz, M. \& Valdés, A. (2005). Cambios en la ocupación de los adultos mayores recientemente jubilados. Revista Chilena de Terapia Ocupacional, (5), 13p. doi: 10.5354/0717-5346.2005.103
Atchley, R. (1975). Adjustment to loss of job at retirement. International Journal of Aging Human Development, 6(1), 17-27. doi: 10.2190/ EHU3-VCRV-VCRJ-04NU

Aymerich, M., Planes, M. \& Gras, M.E. (2010). La adaptación a la jubilación y sus fases: Afectación de los niveles de satisfacción y duración del proceso adaptativo. Anales de Psicología, 26(1), 80-88. Recuperado de http://www.um.es/analesps/v26/v26_1/10-26_1.pdf

Centro Internacional de Longevidad de Brasil. (2015). Envejecimiento activo: Un marco político ante la revolución de la longevidad. Centro Internacional de Longevidade Brasil, (1 ${ }^{\text {a }}$ ed.). Recuperado de https:// www.easp.es/?.wpdmact=process\&did=MTMzLmhvdGxpbms

Christiansen, C.H. (1999). Defining lives: Occupation as identity: An essay on competence, coherence, and the creation of meaning. American Journal of Occupational Therapy, (53), 547-558. doi: 10.5014/ajot.53.6.547

Flick, U. (2015). El diseño de Investigación cualitativa. Madrid: Morata.

Gerrish, K. \& Lacey, A. (2008). Investigación en enfermería, (5 ed.) Madrid: McGraw Hill.

HelpAge International. (2015). Global AgeWatch Index 2015 Insight report. Londres.

Hermida, P.D., Tartaglini, M.F. \& Stefani, D. (2016). Actitudes y significados acerca de la jubilación: un estudio comparativo de acuerdo al género en adultos mayores. Liberabit, 22(1): 57-66. Recuperado de: http://revistaliberabit.com/es/revistas/RLE_22_1_actitudes-y-significados-acerca-de-la-jubilacion-un-estudio-comparativo-de-acuerdo-al-genero-en-adultos-mayores.pdf

Jonsson, H., Josephsson, S. \& Kielhofner, G. (2001). Narratives and experience in an occupational transition: A longitudinal study of the retirement process. American Journal of Occupational Therapy, 55(4), 424-432. doi: 10.5014/ajot.55.4.424

Jonsson, H., Kielhofner, G. \& Borell, L. (1997). Anticipating retirement: The formation of narratives concerning an occupational transition. American Journal of Occupational Therapy, 51(1), 49-56. doi 10.5014/ajot.51.1.49

Leandro, C., Giardini, S. \& Bratfish, M. (2014). Efeitos de uma intervenção breve no planejamento para a aposentadoria. Florianópolis. Revista Psicologia Organizações e Trabalho, 14(3), 257-270. Recuperado de http://pepsic.bvsalud.org/pdf/rpot/v14n3/v14n3a02.pdf

Ley Orgánica 15/1999, de 13 de diciembre, de Protección de Datos de Carácter Personal. Boletín Oficial del Estado de España, número 298, de 14 de diciembre de 1999 .

Ley 27/2011, de 1 de agosto, sobre actualización, adecuación y modernización del sistema de Seguridad Social. Boletín Oficial del Estado de España, número 184, de 2 de agosto de 2011.

Pettican, A., Prior, S. (2011). It's a new way of life: an exploration of the occupational transition of retirement. British Journal of Occupational Therapy, 12-19. Doi /10.4276/030802211X12947686093521.

Price, C.A. (2002). Retirement for women: the impact of employment. Journal of Women and Aging, 14(3-4), 41-57. doi: 10.1300/J074v14n03_04 
Prochaska, J. \& DiClemente, C. (1984). The transtheoretical approach New York: Dow Jones.

Teuscher, U. (2010). Change and persistence of personal identities after the transition to retirement. International Journal of Aging Human Development, 70(1), 89-106. doi: 10.2190/AG.70.1.d

Unión Democrática de Pensionistas y Jubilados de España, IMSERSO. (1990). Jornadas internacionales 26 y 27 de Noviembre: Preparación para la jubilación. Madrid.

United Nations. (2015). World Population Aging 2015. New York: Department of Economic and Social Affairs Population Division. Serie de Informes Técnicos: 390

Vianna, C. \& Melo-Silva, L.L. (2009). Expectativas diante da aposentadoria Um estudo de acompanhamento em momento de transiçao. Revista Brasileira de Orientação Profissional, 10(1), 45-54. Recuperado de: http://pepsic.bvsalud.org/scielo.php?script=sci_arttext\&pid=S167933902009000100007

\section{TABLAS}

Tabla I: Criterios de inclusión y exclusión.

\begin{tabular}{|l|l|l|}
\hline \multirow{2}{*}{ CRITERIOS DE INCLUSIÓN } & $\checkmark$ & Residir en el área metropolitana da Coruña. \\
& $\checkmark$ Estar jubilado desde hace un máximo de 7 años. \\
& $\checkmark$ Acudir a las entidades seleccionadas para la entrada al campo. \\
& $\checkmark$ Manifestar interés para participar en el estudio. \\
\hline CRITERIOS DE EXCLUSIÓN & $\times$ Haber pasado la mayor parte de su vida en entorno rural. \\
& $\times$ No haber tenido un puesto de trabajo remunerado. \\
& $\times$ \\
& \\
\hline
\end{tabular}

\section{FIGURAS}

Figura 1: Asociación entre tipo de jubilación y sexo.

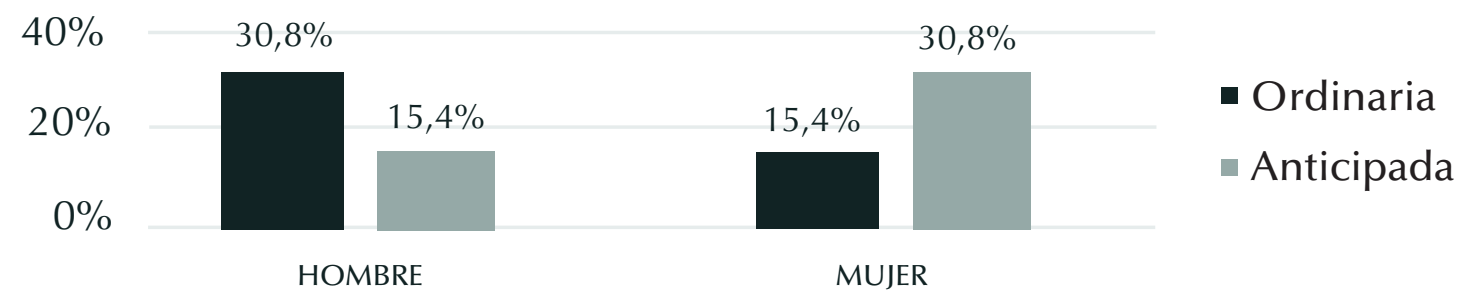


Figura 2: Asociación entre tipo de jubilación y problemas de salud.

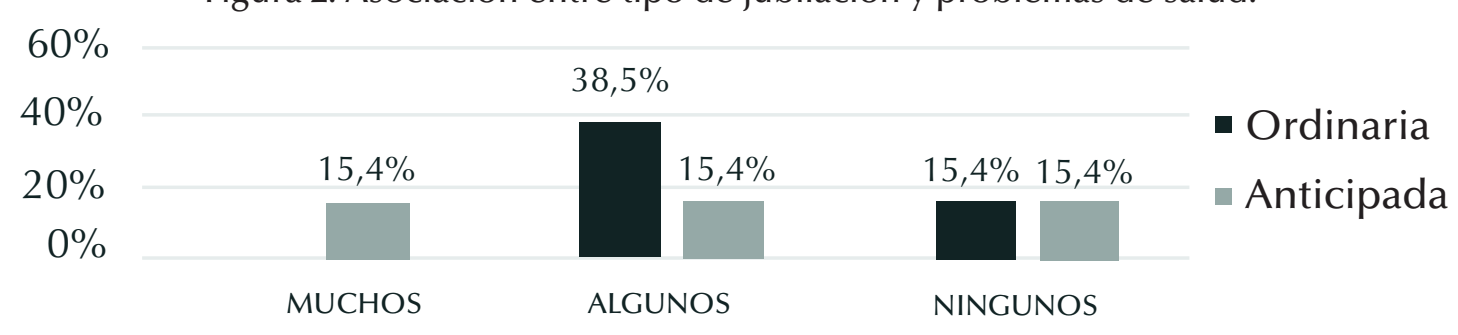

Figura 3: Relación entre satisfacción con la distribución del tiempo y calidad de vida.

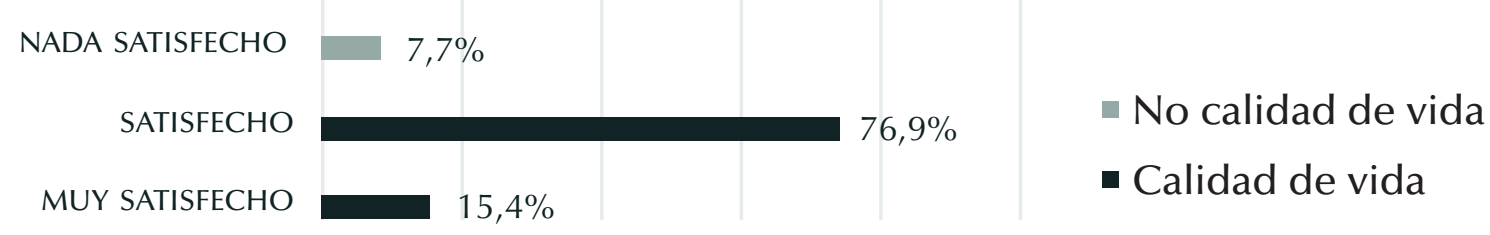

$\begin{array}{llllll}0 \% & 20 \% & 40 \% & 60 \% & 80 \% & 100 \%\end{array}$

Figura 4: Mapa conceptual de resultados de la investigación cualitativa.

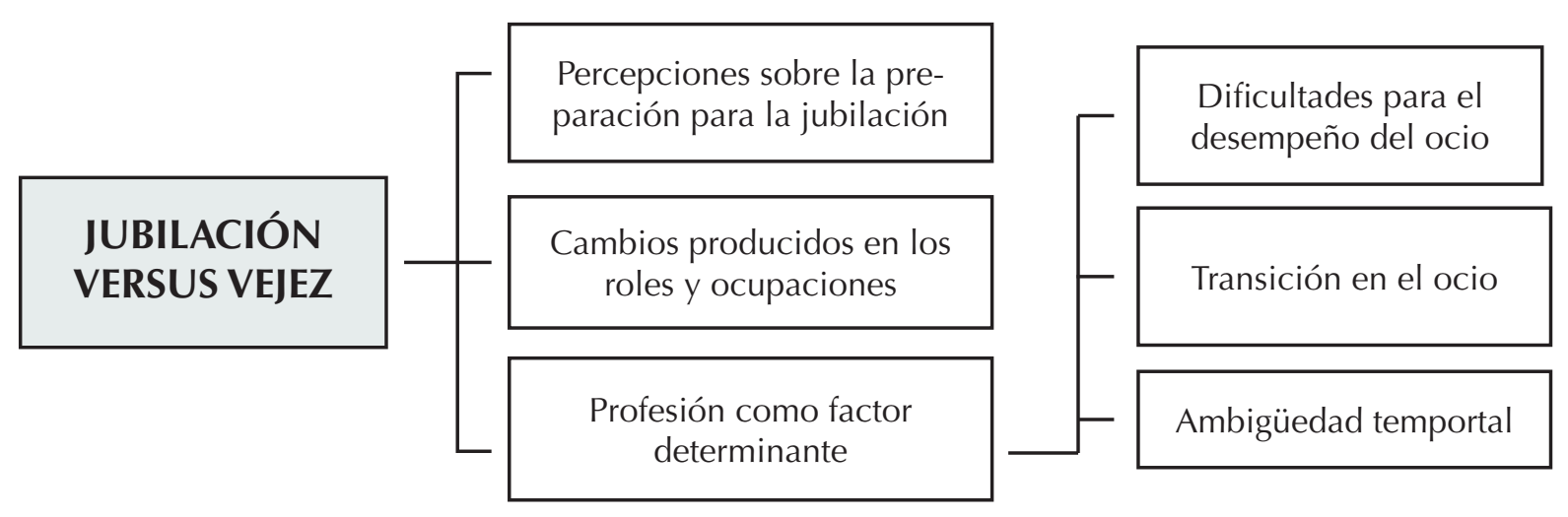




\section{APÉNDICES}

\section{Apéndice 1: Cuestionario}

A continuación cubra el cuestionario indicando con una cruz cuál es su situación personal. En caso de no tener opciones a elegir escriba lo que corresponda.

Participante:

Edad:

Sexo:

$\square$ Masculino

$\square \quad$ Femenino

Estado civil:

$\square$ Soltero/a

$\square$ Casado/a

$\square$ Pareja de hecho

$\square$ Divorciado/a

$\square$ Viudo/a

$\square$ Separado/a

Nivel educativo:

Sin estudios

$\square \quad$ Estudios básicos

Educación secundaria

$\square$ Bachillerato

$\square$ Estudios universitarios

Carácter de la jubilación:

Voluntaria

$\square$ Forzosa

Tipo de jubilación:

$\square$ Ordinaria

anticipada

$\square$ Parcial

Profesión principal:

Tiempo de duración de la vida laboral:

Tiempo que ha transcurrido tras la jubilación
Grado de satisfacción con la etapa laboral:

$\square$ Nada satisfecho/a

Algo satisfecho/a

Ni satisfecho/a ni insatisfecho/a

$\square$ Satisfecho/a

$\square$ Muy satisfecho/a

¿A qué actividades dedica más tiempo?

Trabajo

$\square$ Ocio y tiempo libre

$\square$ Descanso y sueño

$\square$ Cuidado de otros

$\square$ Cuidado personal

Participación en la comunidad

¿A qué actividades dedica menos tiempo?

$\square$ Trabajo

$\square$ Ocio y tiempo libre

$\square$ Descanso y sueño

$\square$ Cuidado de otros

$\square$ Cuidado personal

$\square$ Participación en la comunidad

Actualmente, ise siente satisfecho/a con la distribución de su tiempo con respecto a sus actividades cotidianas? $\square$ Nada satisfecho/a

$\square$ Algo satisfecho/a

Ni satisfecho/a ni insatisfecho/a

$\square$ Satisfecho/a

- Muy satisfecho/a

¿Ha realizado una preparación previa a la jubilación? $\square$ Sí

$\square \mathrm{No}$

¿Considera que en la actualidad tiene una buena calidad de vida?

Sí

$\square \quad$ No

En relación con su calidad de vida, ¿tiene problemas de salud?

Muchos

$\square \quad$ Algunos

Ningunos 
Finalmente, ¿cómo considera que ha sido su jubilación?

$\square$ Positiva

$\square \quad$ Ni positiva ni negativa

$\square \quad$ Negativa

\section{Apéndice 2: Guion de entrevista}

\section{Bloque I: Etapa previa a la jubilación}

$\rightarrow$ ¿Cuál ha sido su profesión hasta el momento de retirarse?, ¿cómo era un día de trabajo normal para usted?

$\rightarrow$ ¿Cómo eran las relaciones en su ámbito laboral?, ¿qué actividades ocio y tiempo libre desempeñaba?, ¿qué otras actividades Ilevaba a cabo?

\section{Bloque II: Momento de la jubilación}

$\rightarrow$ ¿Cuándo se jubiló?, ¿cómo fue el modo de acceder a la jubilación?

$\rightarrow$ ¿Cómo ha vivido el periodo de transición de la vida laboral al retiro?

$\rightarrow$ ¿Considera necesaria la preparación previa a esta etapa?, ¿por qué?

$\rightarrow$ En su caso, ¿en qué consistió dicha preparación?

$\rightarrow$ ¿Piensa que la jubilación ha influenciado sobre la distribución del tiempo que dedica a sus actividades cotidianas?, ¿cómo?

$\rightarrow$ ¿Considera que finalizar su vida laboral ha afectado a su salud y calidad de vida?, ¿ha repercutido en su entorno próximo (en su familia, amigos...)?

\section{Bloque III: Desempeño ocupacional actual}

$\rightarrow$ Actualmente, ¿cómo es un día normal para usted?

$\rightarrow$ ¿Cómo emplea su tiempo libre?, ¿cómo quisiera emplearlo?

\section{Bloque IV: Expectativas de futuro}

$\rightarrow$ ¿Qué expectativas tenía usted sobre esta etapa de su vida?

$\rightarrow$ ¿Qué proyectos de futuro tiene en mente tras la jubilación?

\section{Bloque V: Reflexiones finales}

$\rightarrow$ En general, ¿qué ha significado la jubilación para usted?

$\rightarrow$ Por último, le vuelvo a preguntar, ¿considera necesaria

la preparación previa a la jubilación? 